\title{
Systematic Review of Quality of Life Assessments after Cochlear Implantation in Older Adults
}

\author{
Ellen Andries ${ }^{a, b} \quad$ Annick Gilles ${ }^{a-c}$ Vedat Topsakal ${ }^{a, b} \quad$ Olivier M. Vanderveken ${ }^{a, b}$ \\ Paul Van de Heyning ${ }^{a, b}$ Vincent Van Rompaey ${ }^{a, b} \quad$ Griet Mertens $^{a, b}$ \\ ${ }^{a}$ Department of Otorhinolaryngology, Head and Neck Surgery, Antwerp University Hospital (UZA), Antwerp, \\ Belgium; ${ }^{b}$ Experimental Laboratory of Translational Neurosciences and Dento-Otolaryngology, Faculty of Medicine \\ and Health Sciences, University of Antwerp (UA), Antwerp, Belgium; ' ${ }^{C}$ Department of Education, Health and Social \\ Work, University College Ghent (HoGent), Ghent, Belgium
}

\section{Keywords}

Older adults · Cochlear implant - Quality of life .

Health-related quality of life · Cost-utility

\begin{abstract}
Cochlear implants $(\mathrm{Cl})$ have increasingly been adopted in older adults with severe to profound sensorineural hearing loss as a result of the growing and aging world population. Consequently, researchers have recently shown great interest in the cost-effectiveness of cochlear implantation and its effect on quality of life (QoL) in older $\mathrm{Cl}$ users. Therefore, a systematic review and critical evaluation of the available literature on QoL in older adult $\mathrm{Cl}$ users was performed according to the Preferred Reporting Items for Systematic Reviews and Meta-Analyses (PRISMA) guidelines. Studies were selected by searching MEDLINE (PubMed) and the Cochrane Library and by checking the reference lists of relevant articles. Inclusion criteria were as follows: (1) the study sample were adults aged 50 years and older with postlingual onset of bilateral severe to profound hearing loss, (2) all subjects received a multi-electrode $\mathrm{Cl}$, and (3) QoL was assessed before
\end{abstract}

and after implantation. Out of 1,093 records, 18 articles were accepted for review. Several studies demonstrated significant positive effects of cochlear implantation on QoL in older adults, but high-level evidence-based medicine is lacking. An improvement of QoL was generally reported when using disease-specific instruments, which are designed to detect treatment-specific changes, whereas the outcomes of generic QoL questionnaires, assessing general health states, were rather ambiguous. However, only generic questionnaires would be able to provide calculations of the cost-effectiveness of $\mathrm{Cl}$ and comparisons across patient populations, diseases, or interventions. Hence, generic and diseasespecific QoL instruments are complementary rather than contradictory. In general, older Cl users' QoL was assessed using a variety of methods and instruments, which complicated comparisons between studies. There is a need for a standardized, multidimensional, and comprehensive QoL study protocol including all relevant generic and diseasespecific instruments to measure and compare QoL, utility, and/or daily life performance in $\mathrm{Cl}$ users.

(c) 2020 S. Karger AG, Basel

$\begin{aligned} & \text { karger@karger.com } \\ & \text { www.karger.com/aud }\end{aligned}$
Karger 2020 S. Karger AG, Basel




\section{Introduction}

Hearing loss prevalence is rising due to the aging world population, as the odds of having a hearing impairment increase with age. According to the World Health Organization (WHO), 1 out of 10 people will have disabling hearing loss in 2050 [WHO, 2018]. Several large studies have demonstrated that hearing impairment, affecting 466 million people worldwide, is associated with a greater risk of cognitive impairment, social isolation, and loneliness [Ciorba et al., 2012; Lin et al., 2013; Sung et al., 2016]. Hearing rehabilitation may provide a therapeutic approach to ameliorating these risks. Hearing aids may be used in the case of mild-to-moderate sensorineural hearing loss (SNHL), while cochlear implantation can rehabilitate hearing in the case of severe-to-profound SNHL. Cochlear implants (CI) have proven their safety in infants and the elderly [Roland et al., 2009; Ramsden et al., 2012; Yang and Cosetti, 2016; Hofkens-Van Den Brandt et al., 2019]. However, since CI is increasingly adopted in older adults, interest in studying its impact on quality of life (QoL), as well as its cost-utility, has grown as these issues are highly linked with CI candidacy and reimbursement criteria [Teschner et al., 2013].

The WHO defines QoL as follows: “Individuals' perceptions of their position in life in the context of the culture and value systems in which they live, and in relation to their goals, expectations, standards and concerns" [WHO, 1998]. Questionnaires are the most common instruments to assess QoL. Most questionnaires used in a healthcare setting measure health-related QoL (HRQoL), instead of holistic QoL, which also includes more general factors such as income, adequacy of housing, and so on [Theofilou, 2013]. Theofilou et al. [2013] define HRQoL as: "Optimum levels of mental, physical, role (e.g., work, parent, carer, etc.) and social functioning, including relationships, and perceptions of health, fitness, life satisfaction and well-being." In this study, no distinction will be made between HRQoL and QoL.

An important outcome to evaluate any intervention, for example a drug or an implanted medical device such as a CI, is the change in the subject's own perceived state of health. This can be studied by evaluating HRQoL, utility (preference-based health state), and daily life performance. Numerous HRQoL measurement instruments have been developed, including generic and disease-specific instruments. Generic measures can be used in various diseases, populations, and interventions as they assess general health states. A limited number, including the EuroQoL (EQ)-5D and the Health Utilities Index (HUI), can be used to measure health utility [Kind, 1996, Horsman et al., 2003]. Utility scores are HRQoL desirability weights indicating the preference for different health states. These scores range from 0 (death) to 1 (perfect health) on an interval scale. Hence, a higher utility score reflects a more preferable health state. Based on these utility scores, the cost-effectiveness of medical interventions can be estimated [Whitehead and Ali, 2010]. Disease-specific instruments, on the other hand, assess particular diagnostic groups and may be more sensitive to potentially important outcome changes [Patrick and Deyo, 1989; Lin et al., 2012]. The lack of specificity of generic HRQoL measures is often used as an argument to justify the use of a disease-specific HRQoL instrument. Whenever a significant improvement is observed on a disease-specific HRQoL outcome measure and no effect on a generic HRQoL outcome measure, one should critically evaluate whether the changes in disease-specific HRQoL are overestimating the estimated effect of an intervention [EUnetHTA, 2015]. Furthermore, in the field of CI, psycho-acoustic outcome measures such as speech recognition and pure-tone audiometry alone do not always reflect the impact on HRQoL [Capretta and Moberly, 2016]. Hence, the aim of this study is to perform a systematic review and critical evaluation of the literature available on HRQoL in older CI recipients.

\section{Methods}

The systematic review was performed according to the Preferred Reporting Items for Systematic Reviews and Meta-Analyses (PRISMA) guidelines [Liberati et al., 2009]. The objective was to critically assess current literature concerning HRQoL in CI recipients.

\section{Eligibility Criteria}

- Participants: the study sample consisted of adults aged 50 years and older with postlingual onset of bilateral severe to profound hearing loss. All subjects received a multi-electrode CI.

- Intervention: Cochlear implantation with a multi-electrode CI.

- Comparator: No restrictions were imposed.

- Outcomes: Changes in self-reported HRQoL scores from baseline to the last available follow-up, measured by generic questionnaires, hearing-specific questionnaires, or questionnaires that did not belong to one of the previous 2 categories.

- Study design: Longitudinal studies with retrospective as well as prospective study designs were eligible; HRQoL had to be measured preoperatively at baseline and at least one measurement had to be performed postoperatively; studies could include a control group.

- Reviews: study protocols and letters to the editor were excluded for full-text review; no restrictions were imposed regarding language, publication date, and publication status.
62

Audiol Neurotol 2021;26:61-75 DOI: $10.1159 / 000508433$
Andries et al. 
Search Strategy

Studies were selected by searching MEDLINE (PubMed) and the Cochrane Library and by checking the reference lists of relevant articles. The literature search started on August 2, 2019 and was completed on August 9, 2019. The Cochrane and MEDLINE search strategies are available in online supplementary Appendix $A$ and B (see www.karger.com/doi/10.1159/000508433 for all online suppl. material). First, the titles and abstracts of the obtained records were screened. Second, full texts of records passing the screening phase were retrieved and analyzed. Two authors (E.A. and G.M.) performed study selection independently. Potential disagreements were resolved by consensus. Corresponding authors were contacted if any of the inclusion criteria of the review were not or not clearly mentioned in the article or if the full-text article was not available.

\section{Information Extraction}

Information extraction from the selected studies included study design, patient characteristics (study sample, age, gender, onset of hearing loss, duration of deafness, hearing aid use, etiology, etc.), measurement timing (preoperatively and postoperatively), HRQoL questionnaires used (name of the questionnaire and questionnaire category: hearing-specific, generic, or other), and results (change in HRQoL score after CI per questionnaire category). Risk of bias was assessed, taking into account Choi and Pak's [2005] catalog of biases in questionnaires. The following 4 potential sources of bias were evaluated: response bias, selection bias, statistical methods, and other risks of bias. Response bias concerns how many subjects completed the questionnaires in proportion to the total amount of subjects having participated in the study. Studies with a response rate of $<80 \%$ are considered to be at risk for response bias. Selection bias occurs when the study sample is not a good representation of the population, for example when only CI users with the highest speech intelligibility scores are included. Statistical methods and other sources of bias were also considered. The latter included sample size, questionnaire administration method, lack of validation of the questionnaires, and the use of proxy respondents. The principal summary measures are the $p$ values of the changes in HRQoL per questionnaire category per study.

\section{Results}

\section{Study Selection}

The PubMed and Cochrane Library search provided 1,085 records, of which 1,046 were retrieved from PubMed and 39 from the Cochrane Library. In addition, 28 records were identified by searching the reference lists of relevant articles. After the removal of duplicates, 1,093 records remained. The abstracts and titles of those records were screened, resulting in the selection of 86 articles for full-text assessment and the exclusion of 1,007 irrelevant articles. Eventually, 18 studies met the inclusion criteria listed above and were accepted for review [Vermeire et al., 2005; Noble et al., 2009; Park et al., 2011; Chung et al., 2012; Zwolan et al., 2014; Mosnier et al.,
2015; Castiglione et al., 2016; Choi et al., 2016; Contrera et al., 2016; Knopke et al., 2016; Olze et al., 2016; Contrera et al., 2017; Sonnet et al., 2017; Mosnier et al., 2018; Völter et al., 2018; Knopke et al., 2019; Sarant et al., 2019].

Out of the included articles, 68 did not meet the inclusion criteria listed above and were excluded for the following reasons. Twenty-two studies only measured HRQoL after implantation, but not before [Kelsall et al., 1995; Shin et al., 1997; Kunimoto et al., 1999; Eshraghi et al., 2009; Ramos et al., 2013; Sanchez-Cuadrado et al., 2013; Cloutier et al., 2014; Huarte et al., 2015; Sladen and Zappler, 2015; Speers et al., 2015; Aimoni et al., 2016; Alhanbali et al., 2016; Manrique-Huarte et al., 2016; Ramos-Macias et al., 2016; Hinder et al., 2017]. They were excluded because only studies assessing changes in HRQoL were accepted for review. Studies planning their preoperative HRQoL measurement postimplantation, for example by sending questionnaires about HRQoL before CI after implantation, were also excluded [Djalilian et al., 1999; Shin et al., 2000; Francis et al., 2002; Cohen et al., 2004; Olze et al., 2012; Di Nardo et al., 2014; Tang et al., 2017]. Participants younger than 50 years were included in 28 studies [Maillet et al., 1995; Summerfield and Marshall, 1995; Knutson et al., 1998; Palmer et al., 1999; Hamzavi et al., 2001; Padilla Romero et al., 2004; Mo et al., 2005; Damen et al., 2007; Noble et al., 2008; Pan et al., 2009; Olze et al., 2011; Olze et al., 2012; Lenarz et al., 2013; Arnoldner et al., 2014a, b; Francis et al., 2015; Rösli et al., 2015; Rumeau et al., 2015; Sanchez-Cuadrado et al., 2015; Derks et al., 2016; Ottaviani et al., 2016; Ramakers et al., 2016; Runge et al., 2016; Jayakody et al., 2017; Kelsall et al., 2017; Ambert-Dahan et al., 2018; Roland et al., 2018; Summerfield and Barton, 2019]. The combination of participants younger than 50 years and HRQoL measurement only after CI occurred 6 times [Hirschfelder et al., 2008; Rembar et al., 2009; Fuller et al., 2012; Angelo et al., 2016; Calvino et al., 2016; Capretta and Moberly, 2016]. The full texts of 2 studies were not available. Corresponding authors were contacted but did not provide the full-text article. Measurements were not defined as HRQoL in 2 of the included articles. Waltzman et al. [1993] did not assess HRQoL with standardized questionnaires and Mosnier et al. [2015] only assessed telephone use, which cannot represent the daily-life functioning of participants. Two study protocols and 3 reviews were also excluded [Ciorba et al., 2012; Semenov et al., 2012; Cherko et al., 2016; Claes et al., 2016; Rapport et al., 2018]. Single-channel CIs were used in one study and another study only measured HRQoL preoperatively and not postoperatively [Cooper et al., 1989; Sung et 


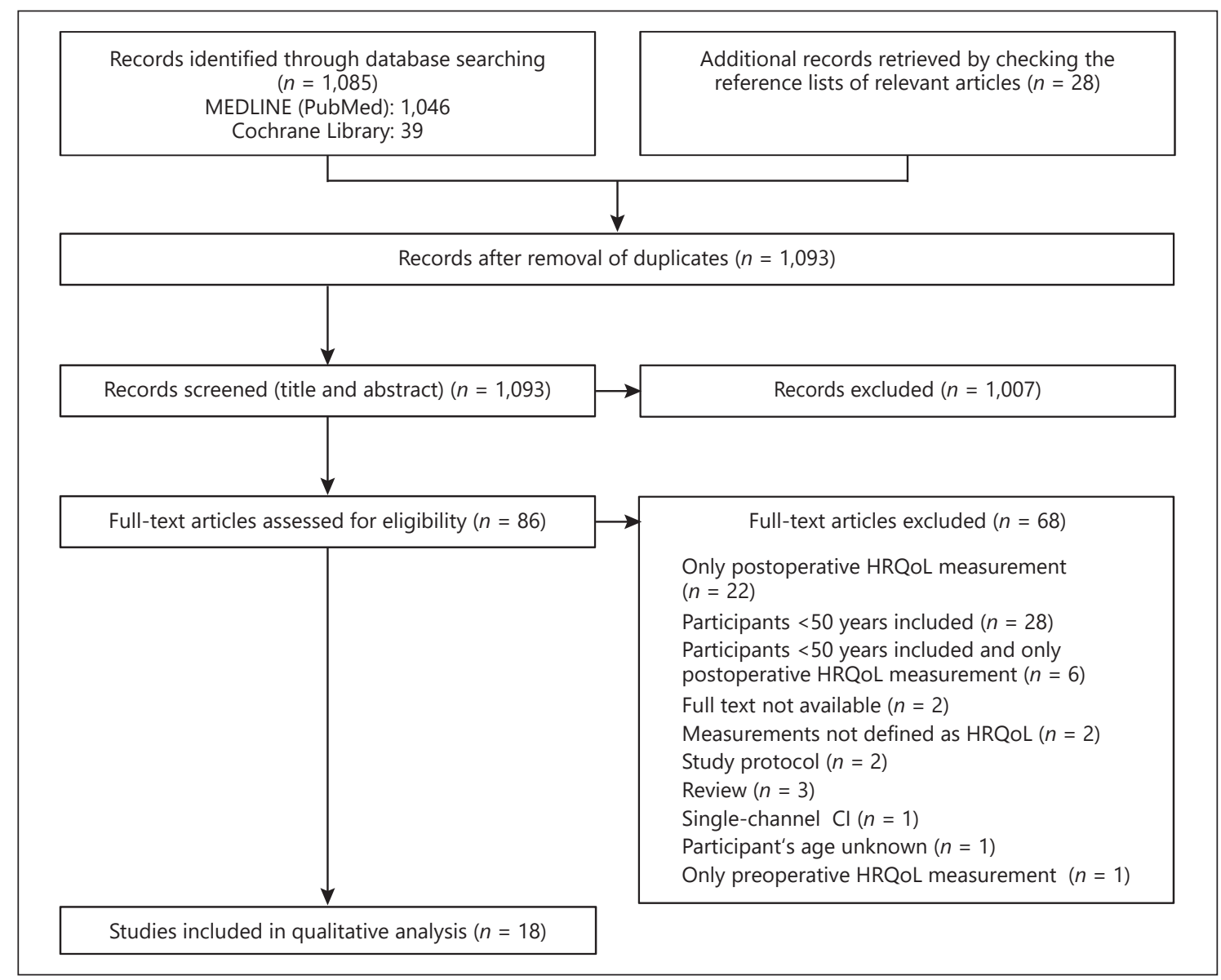

Fig. 1. Flowchart of the selection process.

al., 2016]. One study was removed because the age of the participants was not clearly mentioned in the article [Mick et al., 2014]. The corresponding author was contacted but could not provide any further data than what was published. Figure 1 shows a flowchart of the selection process.

\section{Information Extraction}

In accordance with the inclusion criteria, all studies accepted for review assessed HRQoL pre- and postoperatively in severely hearing-impaired older CI users. Out of the 18 included articles, 15 were prospective longitudinal cohort studies. Furthermore, the study by Park et al. [2011] was a retrospective assessment of older CI recipients. Vermeire et al. [2005] conducted a cross-sectional study in which preoperative data were retrieved retrospectively. Zwolan et al. [2014] retrieved data collected during a multicenter prospective study and analyzed it post hoc.
Table 1 provides an overview of the included studies. The full text of all studies was published in English and the year of publication ranged between 2005 and 2019. Participants' HRQoL was followed-up for at least 3 months to a maximum of 7 years post-CI. HRQoL was assessed at baseline and 1 or 2 HRQoL measurements took place after implantation. Nine studies additionally included separate groups of subjects not fully meeting the eligibility criteria of the review. These subject groups were not included in the outcome analysis of the review. Overall, the selected studies involved 925 subjects meeting the inclusion criteria of the review and 1,514 subjects in total. Out of the subjects meeting the inclusion criteria, $46 \%$ were male and 54\% was female. Four studies did not report gender distribution and were not included in the calculation. The eligible participants' mean age was 71.6 years. Only the median age was given in the study by Castiglione et al. [2016] so the study could not be included in the calculation. 
Table 1. Overview of the included studies

\begin{tabular}{|c|c|c|c|c|c|c|c|c|}
\hline \multirow[t]{2}{*}{ Study } & \multirow{2}{*}{$\begin{array}{l}\text { CI } 50+{ }^{\mathrm{a}}, \\
n\end{array}$} & \multirow{2}{*}{$\begin{array}{l}\text { Others }{ }^{\mathrm{b}} \text {, } \\
n\end{array}$} & \multirow{2}{*}{$\begin{array}{l}\text { Male, } \\
n(\%)\end{array}$} & \multirow{2}{*}{$\begin{array}{l}\text { Female, } \\
n(\%)\end{array}$} & \multirow{2}{*}{$\begin{array}{l}\text { Age, years [mean } \\
\text { (SD) or median] }\end{array}$} & \multicolumn{3}{|c|}{ Evaluation time points } \\
\hline & & & & & & pre-CI & $6 \mathrm{M}$ post-CI & $12 \mathrm{M}$ post-CI \\
\hline Vermeire et al. [2005] & 56 & $33^{\mathrm{c}}$ & N/A & N/A & $68(\mathrm{~N} / \mathrm{A})$ & $\checkmark$ & $\sqrt{ }^{1}$ & - \\
\hline Noble et al. [2009] & 17 & $31^{\mathrm{d}}$ & $5(29.4)$ & $12(70.6)$ & $69.4(5.95)$ & $\checkmark$ & - & $\sqrt{ }^{\mathrm{m}}$ \\
\hline Park et al. [2011] & 100 & $61^{\mathrm{e}}$ & $39(39)$ & $61(61)$ & $66(\mathrm{~N} / \mathrm{A})$ & $\checkmark$ & - & $\checkmark$ \\
\hline Chung et al. [2012] & 68 & $215^{\mathrm{f}}$ & N/A & N/A & $71.53(4.36)$ & $\checkmark$ & - & $\checkmark$ \\
\hline Zwolan et al. [2014] & 18 & $20^{\mathrm{g}}$ & $11(61)$ & $7(39)$ & $74.78(6.65)$ & $\checkmark$ & $\checkmark$ & $\checkmark$ \\
\hline Mosnier et al. [2015] & 94 & $\mathrm{~N} / \mathrm{A}$ & $45(47.9)$ & $49(52.1)$ & $72(5.0)$ & $\checkmark$ & $\checkmark$ & $\checkmark$ \\
\hline Castiglione et al. [2016] & 15 & $110^{\mathrm{h}}$ & $8(53)$ & $7(47)$ & 71 & $\checkmark$ & - & $\checkmark$ \\
\hline Choi et al. [2016] & 50 & $63^{i}$ & $29(58)$ & $21(42)$ & $69.2(\mathrm{~N} / \mathrm{A})$ & $\checkmark$ & $\checkmark$ & $\checkmark$ \\
\hline Contrera et al. [2016] & 50 & $63^{\mathrm{i}}$ & $29(58)$ & $21(42)$ & $69.2(\mathrm{~N} / \mathrm{A})$ & $\checkmark$ & $\checkmark$ & $\checkmark$ \\
\hline Knopke et al. [2016] & 17 & $\mathrm{~N} / \mathrm{A}$ & $\mathrm{N} / \mathrm{A}$ & N/A & $82.9(2.7)$ & $\checkmark$ & $\checkmark$ & - \\
\hline Olze et al. [2016] & 79 & $\mathrm{~N} / \mathrm{A}$ & $35(44.3)$ & $44(55.7)$ & $75.4(\mathrm{~N} / \mathrm{A})$ & $\checkmark$ & $\checkmark$ & - \\
\hline Contrera et al. [2017] & 50 & $63^{\mathrm{i}}$ & $29(58)$ & $21(42)$ & $69.2(\mathrm{~N} / \mathrm{A})$ & $\checkmark$ & $\checkmark$ & $\checkmark$ \\
\hline Sonnet et al. [2017] & 16 & $\mathrm{~N} / \mathrm{A}$ & $6(37.5)$ & $10(62.5)$ & $72.5(\mathrm{~N} / \mathrm{A})$ & $\checkmark$ & $\checkmark$ & $\checkmark$ \\
\hline Claes et al. [2018] & 20 & N/A & $12(60)$ & $8(40)$ & $71.49(8.05)$ & $\checkmark$ & $\checkmark$ & $\checkmark$ \\
\hline Mosnier et al. [2018] & 70 & $\mathrm{~N} / \mathrm{A}$ & $27(38.6)$ & $43(61.4)$ & $72(5.1)$ & $\checkmark$ & - & $\sqrt{ }^{\mathrm{n}}$ \\
\hline Völter et al. [2018] & $60^{j}$ & N/A & N/A & N/A & $65.8(8.9)$ & $\checkmark$ & $\checkmark$ & $\checkmark$ \\
\hline Knopke et al. [2019] & 86 & N/A & $45(52.3)$ & $41(47.7)$ & $76.21(5.33)$ & $\checkmark$ & $\checkmark$ & $\checkmark$ \\
\hline Sarant et al. [2019] & $59^{\mathrm{k}}$ & $\mathrm{N} / \mathrm{A}$ & $36(61)$ & $23(39)$ & $72.3(6.8)$ & $\checkmark$ & - & $\sqrt{ }^{\mathrm{o}}$ \\
\hline
\end{tabular}

$\checkmark$, evaluation took place in the study; -, evaluation did not take place in the study; M, months; N/A, not available.

a Number of CI patients aged 50 years and older included in the study.

${ }^{\mathrm{b}}$ Number of participants included in the study besides CI patients of 50 years and older.

${ }^{c} \mathrm{CI}$ recipients were divided into 3 age groups: younger than 55, 56-69, and 70 years and older. The number of patients aged 56-69 years and 70 years and older is given in CI 50+, the number of patients aged younger than 55 years is given in Others.

${ }^{\mathrm{d}}$ First, participants were divided into 3 groups regarding their CI configuration: $\mathrm{CI}$ and hearing aid, CI only, and bilateral CI. These groups were then divided into patients older than 60 years and patients younger than 60 years. The number of CI-only and $\mathrm{CI}$ and hearing aid patients older than 60 years is given in CI 50+, the number of the other patients with pre- and postoperative measurements is given in Others.

${ }^{\mathrm{e}} \mathrm{CI}$ recipients were divided into 3 age groups: 50 years and younger, 51-65 years, and older than 65 years. Additionally, they were divided based on prior hearing aid use: none, binaural hearing aid, monaural hearing aid. The number of patients aged 5165 and older than 65 years, regardless of prior hearing aid use, is given in CI 50+, the number 50 years and younger, regardless of prior hearing aid use, is given in Others.

${ }^{\mathrm{f}} \mathrm{CI}$ users were divided into 3 age groups: younger than 45 years, $45-65$ years, and older than 65 years. The number of patients older than 65 years is given in CI $50+$, the number of other patients is given in Others.

${ }^{g}$ CI users were divided into 2 age groups: younger than 65 and older than 65 years. The number of patients older than 65 years is given in CI $50+$, the number of patients younger than 65 years is given in Others.

$\mathrm{h}$ Twenty participants with normal hearing, thirty 1-month bilateral hearing aid users, 30 experienced bilateral hearing aid users, 15 experienced unilateral hearing aid users, and 15 participants with mild to moderate hearing loss without treatment were included in the study; all older than 65 years.

${ }^{\mathrm{i}}$ The number of participants aged 50 years or older with hearing aids.

${ }^{j}$ At baseline, 60 patients were assessed; out of these, 33 patients were evaluated 6 months postoperatively and 20 patients underwent testing 6 and 12 months postoperatively.

${ }^{\mathrm{k}}$ At baseline, 59 patients were assessed; out of these, 20 patients were evaluated 18 months postoperatively.

${ }^{1}$ Postoperative measurement timing varied from 3 months post-CI to 36 months post-CI.

${ }^{\mathrm{m}}$ Postoperative measurement timing was at least 12 months.

${ }^{\mathrm{n}}$ Participants were additionally followed up 7 years post-CI.

${ }^{\circ}$ Participants were assessed 18 months postoperatively.

\section{HRQoL Outcomes}

A list of abbreviations of the included questionnaires is presented in Table 2. An overview of the disease-specific and generic outcomes per subdomain per study is shown in Tables 3 and 4 . More information about the questionnaires is provided in online supplementary Appendix C.

Quality of Life after Cochlear

Implantation in Older Adults

\section{Disease-Specific Outcomes}

All studies using disease-specific, or in this case hearing-specific questionnaires indicated an improvement of disease-specific HRQoL postoperatively. Studies using the Nijmegen Cochlear Implant Questionnaire (NCIQ) indicated a significant improvement in all domains 6 months postoperatively $(p<0.01)$ and scores remained 
Table 2. List of abbreviations of the included questionnaires

\begin{tabular}{ll}
\hline ADS-L & Depression Scale \\
APHAB & Abbreviated Profile of Hearing Aid Benefit \\
B-ADL & Bayer Activities of Daily Living Scale \\
GAD-7 & General Anxiety Disorder-7 \\
GDS & Geriatric Depression Scale \\
HADS & The Hospital Anxiety and Depression Scale \\
HDRS & Hamilton Depression Rating Scale \\
HHI-A & Hearing Handicap Inventory for Adults \\
HHI-E & Hearing Handicap Inventory for the Elderly \\
HHQ & Hearing Handicap Questionnaire \\
HISQUI-19 & Hearing Implant Sound Quality Index 19 \\
HUI-3 & Health Utilities Index \\
I-ADL & Instrumental Activities of Daily Living \\
IPAQ & International Physical Activity Questionnaire \\
Loneliness Scale & Loneliness Scale \\
LSNS & Lubben Social Network Scale \\
NCIQ & Nijmegen Cochlear Implant Questionnaire \\
OI & Oldenburg Inventory \\
PSQ-30 & Perceived Stress Questionnaire \\
SF-36 & Medical Outcome Study Short Form-36 \\
SSQ(12) & Speech Spatial and Qualities of Hearing Scale \\
WHOQOL-OLD & World Health Organization Quality of Life \\
& Assessment for Elderly People \\
\hline
\end{tabular}

stable at 12 months postoperatively [Noble et al., 2009; Mosnier et al., 2015; Knopke et al., 2016; Olze et al., 2016; Claes et al., 2018; Mosnier et al., 2018; Völter et al., 2018; Knopke et al., 2019]. Only the speech production subdomain score did not improve significantly in all studies [Knopke et al., 2016]. In addition, Park et al. [2009] indicated a significant improvement on the Hearing Handicap Inventory for the Elderly (HHI-E) 12 months postoperatively in the emotional and social handicap subscales $(p<0.01)$. No significant difference was observed between the HHI-E scores of younger and older CI recipients. Furthermore, a significant improvement on the Hearing Handicap Inventory for Adults was demonstrated pre- and postoperatively $(p<0.001)$ [Vermeire et al., 2005]. The scores on the emotional $(p=0.003)$ and situational subscale $(p<0.001)$ differed significantly from baseline to postoperatively.

\section{Sound Quality and Speech Understanding Outcomes}

Instruments related to sound quality and speech understanding showed a significant improvement after cochlear implantation. Claes et al. [2018] found that Speech, Spatial and Qualities of Hearing Scale (SSQ12) scores improved significantly at 6 months postoperatively $(p<$ $0.0001)$, and remained stable afterwards $(p=1)$. In addition, a significant improvement 6 months postoperative- ly was indicated, but no additional significant improvement was observed 12 months postoperatively with the Hearing Implant Sound Quality Index 19 (HISQUI-19) [Claes et al., 2018]. Oldenburg Inventory scores also improved significantly after implantation $(p<0.01)$ [Knopke et al., 2016; Olze et al., 2016; Knopke et al., 2019]. Noble et al. [2009] reported that Hearing Handicap Questionnaire (HHQ) scores, SSQ scores, and HHI-A scores of elderly CI users improved postoperatively. However, they did not mention if the improvement in HRQoL was significant. Finally, Abbreviated Profile of Hearing Aid Benefit (APHAB) scores improved significantly $(p<0.001)$ 18 months postoperatively [Sarant et al., 2019].

\section{Generic Outcomes}

Overall, outcomes of studies assessing HRQoL with generic questionnaires were mixed. Studies using the Medical Outcome Study Short Form-36 (SF-36) indicated a significant improvement of social functioning and mental health, but no significant improvement of physical functioning [Chung et al., 2012; Contrera et al., 2016]. In addition, Contrera et al. [2016] found a significant increase in emotional well-being 6 months $(p=0.034)$ and 12 months $(p=0.005)$ after CI, similar to the findings of Chung et al. [2012]. Contrera et al. [2016] also indicated that the Mental Component Summary (MCS) score (comprising vitality, social function, role limitations due to emotional problems, and general mental health perception domains) significantly increased from baseline to 6 months $(p<0.001)$ and 12 months postoperatively. Völter et al. [2018] indicated a significant improvement of the overall WHO Quality of Life Assessment for elderly people (WHOQOL-OLD) score $(p=0.027) 6$ months postoperatively. Sensory functions $(p<0.001)$ and autonomy-related questions $(p=0.026)$ scores also improved significantly, but there was no further improvement at 12 months postoperatively. In contrast, Sonnet et al. [2017] only showed a significant improvement in sensory abilities $(p<0.05) 6$ and 12 months postoperatively.

\section{Utility Outcomes}

An overview of the utility outcomes can be found in Table 5 and a list of abbreviations of the included questionnaires is presented in Table 2. More information about these questionnaires is given in online supplementary Appendix C. There were notable differences in results between studies assessing utility. Zwolan et al. [2014] demonstrated a significant improvement postoperatively in HUI-3 hearing ( $p<0.001)$, but not in HUI-3 multiattribute scores. On the other hand, only female subjects 
Table 3. Disease-specific HRQoL outcomes

\begin{tabular}{|c|c|c|c|c|c|c|c|c|c|c|c|}
\hline & $\begin{array}{l}\text { Vermeire } \\
\text { et al. [2005] } \\
(n=56)\end{array}$ & $\begin{array}{l}\text { Noble } \\
\text { et al. [2009] } \\
(n=17)\end{array}$ & $\begin{array}{l}\text { Park } \\
\text { et al. [2011] } \\
(n=100)\end{array}$ & $\begin{array}{l}\text { Mosnier } \\
\text { et al. [2015] } \\
(n=94)\end{array}$ & $\begin{array}{l}\text { Mosnier } \\
\text { et al. [2018] } \\
(n=70)\end{array}$ & $\begin{array}{l}\text { Knopke } \\
\text { et al. [2016] } \\
(n=17)\end{array}$ & $\begin{array}{l}\text { Knopke } \\
\text { et al. [2019] } \\
(n=86)\end{array}$ & $\begin{array}{l}\text { Olze et al. } \\
{[2016]} \\
(n=79)\end{array}$ & $\begin{array}{l}\text { Claes } \\
\text { et al. [2018] } \\
(n=20)\end{array}$ & $\begin{array}{l}\text { Völter } \\
\text { et al. [2018] } \\
\left(n=60^{\mathrm{a}}\right)\end{array}$ & $\begin{array}{l}\text { Sarant } \\
\text { et al. [2019] } \\
\left(n=59^{\mathrm{b}}\right)\end{array}$ \\
\hline \multicolumn{12}{|l|}{ NCIQ } \\
\hline Basic sound perception & & & & $* *$, & $* *$, & ** & $* *$, & ** & $* * * *$, & $* * *$, & \\
\hline Advanced sound perception & & & & $* *$, & $* *$, & ** & $* *$, & $* *$ & $* * * *$, & $* * *$, & \\
\hline Speech production & & & & $* *$, & $* *$, & ns & $* *, \downarrow$ & ** & $* * * *$, & $* * *$, & \\
\hline Self-esteem & & & & $* *$, & $* *$, & ** & $*$, & ** & $* * * *$, & $* * *$ & \\
\hline Activity & & & & $* *$, & $* *$, & ** & $* *$, & $* *$ & $* * * *$, & $* * *$ & \\
\hline Social interactions & & & & $* *$, & $* *$, & $* *$ & $* *$, & $* *$ & $* * * *$, & $* * *$ & \\
\hline Total & & & & $* *$, & $* *$, & $* *$ & $* *$, & ** & $* * * *$, & $* * *$ & \\
\hline \multicolumn{12}{|l|}{ HHI-E } \\
\hline Emotional subscale & & $?$ & ** & & & & & & & & \\
\hline Social/situational subscale & & $?$ & $* *$ & & & & & & & & \\
\hline \multicolumn{12}{|l|}{ HHI-A } \\
\hline Emotional subscale & ** & & & & & & & & & & \\
\hline Social/situational subscale & $* * *$ & & & & & & & & & & \\
\hline \multicolumn{12}{|l|}{ HHQ } \\
\hline Emotional distress & & $?$ & & & & & & & & & \\
\hline Social restriction & & $?$ & & & & & & & & & \\
\hline \multicolumn{12}{|l|}{$\operatorname{SSQ}(12)$} \\
\hline Speech & & $?$ & & & & & & & $* * * *$, & & \\
\hline Spatial & & ? & & & & & & & $* * * *$, & & \\
\hline Qualities & & $?$ & & & & & & & $* * * *$, & & \\
\hline \multicolumn{12}{|l|}{ OI } \\
\hline Quiet & & & & & & ** & $* *, \$ / * *, \cup, c$ & ** & & & \\
\hline Noise & & & & & & ** & $* *, \varangle / * *, \cup, c$ & ** & & & \\
\hline Localization & & & & & & $* *$ & $* *,{ }^{* *}, \cup,{ }^{c}$ & $* *$ & & & \\
\hline Total & & & & & & $* *$ & $* *,{ }^{* *}, \cup,{ }^{c}$ & $* *$ & & & \\
\hline \multicolumn{12}{|l|}{ HISQUI-19 } \\
\hline Total & & & & & & & & & ****, & & \\
\hline \multicolumn{12}{|l|}{ АРНAB } \\
\hline Ease of communication & & & & & & & & & & & $* * * *$ \\
\hline Reverberation & & & & & & & & & & & $* * * *$ \\
\hline Background noise & & & & & & & & & & & $* * * *$ \\
\hline Aversiveness & & & & & & & & & & & $* * * *$ \\
\hline Global & & & & & & & & & & & $* * * *$ \\
\hline 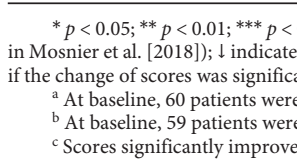 & $\begin{array}{l}.001 ; * * * * p< \\
\text { the outcome } \\
\text { nt; } \cup \text { indicates } \\
\text { assessed. Out } \\
\text { assessed. Out } \\
\text { in the group }\end{array}$ & $\begin{array}{l}.0001 . \mathrm{ns}, \text { not } \\
\text { ecreased to the } \\
\text { the score } 12 \mathrm{~m} \\
\text { f these, } 33 \text { pat } \\
\text { f these, } 20 \text { pat } \\
\text { ged } 70-80 \text { yea }\end{array}$ & $\begin{array}{l}\text { gnificant }(p>c \\
\text { nitial value at } \\
\text { onths postoper } \\
\text { ints were evalu } \\
\text { nts were evalu } \\
\text {, while scores }\end{array}$ & $\begin{array}{l}.05) \text { indicat } \\
2 \text { months post } \\
\text { tively did not } \\
\text { ted } 6 \text { months } \\
\text { ted } 18 \text { month } \\
12 \text { months afte }\end{array}$ & $\begin{array}{l}\text { s the outcome } \\
\text { peratively; gra } \\
\text { gnificantly dif } \\
\text { ostoperatively } \\
\text { postoperativel } \\
\text { implantation }\end{array}$ & $\begin{array}{l}\text { mained stable } \\
\text { hading indicat } \\
\text { from the preo } \\
\text { d } 20 \text { patients }\end{array}$ & $\begin{array}{l}\text { etween } 6 \text { and } 12 \\
\text { serative score. } \\
\text { nderwent testing } \\
\text { ificantly from th }\end{array}$ & $\begin{array}{l}\text { nonths post } \\
\text { indicates an } \\
6 \text { months an } \\
\text { preoperativ }\end{array}$ & $\begin{array}{l}\text { peratively (or } \\
\text { improvement } \\
12 \text { months pc } \\
\text { scores in the } 8\end{array}$ & $\begin{array}{l}\text { veen } 12 \text { month } \\
\text { observed, but } \\
\text { peratively. } \\
\text { group. }\end{array}$ & $\begin{array}{l}\text { ad } 7 \text { years pos } \\
\text { as not menti }\end{array}$ \\
\hline
\end{tabular}

showed a significant improvement of HUI-3 hearing ( $p=$ $0.005)$ and HUI-3 multi-attribute $(p=0.041)$ scores in the study by Sarant et al. [2019].

\section{Daily Living Performance Outcomes}

An overview of the mental well-being and everyday activities outcomes per subdomain per study is presented in Table 6. A list of abbreviations of the included questionnaires can be found in Table 2. In online supplementary Appendix C, more information about the questionnaires is provided.

\section{Mental Well-Being Outcomes}

Results from studies assessing mental well-being were mostly inconsistent. Geriatric Depression Scale (GDS) scores improved 6 months after implantation, which persisted at 12 months after implantation. The improvement was significant in the studies by Choi et al. [2016] $(p<0.05)$ and Castiglione et al. [2016] ( $p<0.01)$, but Mosnier et al.
[2015] did not report a significant improvement. A significant decrease in anxiety $(p=0.002)$ and depression scores $(p=0.002)$ of the Hospital Anxiety and Depression Scale (HADS) was observed 6 months postoperatively [Claes et al., 2018]. However, the results at 12 months postoperatively did not differ significantly from the scores preoperatively and 6 months postoperatively. On the other hand, no significant improvements of General Anxiety Disorder-7 (GAD-7) anxiety scores and Depression Scale (ADS-L) depressiveness scores after implantation were found in the studies by Knopke et al. [2016, 2019]. The mean anxiety and depression level was low pre- and postoperatively in both studies. In addition Knopke et al. [2019] found that there was a significant negative correlation between the ADSL depressiveness score and HRQoL (measured by the NCIQ). Sonnet et al. [2017] used the Hamilton Depression Rating Scale (HDRS). They demonstrated that depressive symptoms of older CI users decreased after implantation, but the decrease was not significant. 
Table 4. Generic HRQoL outcomes

\begin{tabular}{|c|c|c|c|c|}
\hline & $\begin{array}{l}\text { Chung et al. } \\
{[2012](n=68)}\end{array}$ & $\begin{array}{l}\text { Contrera et al. } \\
{[2016](n=50)}\end{array}$ & $\begin{array}{l}\text { Sonnet et al. } \\
{[2017](n=16)}\end{array}$ & $\begin{array}{l}\text { Völter et al. } \\
{[2018]\left(n=60^{\mathrm{a}}\right)}\end{array}$ \\
\hline \multicolumn{5}{|l|}{ SF-36 } \\
\hline \multicolumn{5}{|l|}{ PCS } \\
\hline Physical functioning & ns & \multirow{4}{*}{ ns } & & \\
\hline Role limitations (physical) & ns & & & \\
\hline Bodily pain & ns & & & \\
\hline General health perceptions & ns & & & \\
\hline \multicolumn{5}{|l|}{ MCS } \\
\hline General mental health & $*$ & \multirow{4}{*}{$* *$} & & \\
\hline Social functioning & $*$ & & & \\
\hline Role limitations (emotional) & ns & & & \\
\hline Vitality & ns & & & \\
\hline \multicolumn{5}{|l|}{ WHOQOL-OLD } \\
\hline Sensory abilities & & & $*, \Delta$ & **, \\
\hline Autonomy & & & ns & $*$, \\
\hline Past, present, and future activities & & & ns & ns \\
\hline Social participation & & & ns & ns \\
\hline Death and dying & & & ns & $\mathrm{N} / \mathrm{A}$ \\
\hline Intimacy & & & ns & ns \\
\hline Total & & & ns & $*, \Delta$ \\
\hline
\end{tabular}

${ }^{*} p<0.05$; ** $p<0.01$. ns, not significant $(p>0.05)$; N/A, not available. indicates the outcome remained stable between 6 and 12 months postoperatively; gray shading indicates not applicable.

${ }^{a}$ At baseline, 60 patients were assessed. Out of these, 33 patients were evaluated 6 months postoperatively and 20 patients underwent testing 6 and 12 months postoperatively.

Table 5. Utility outcomes

\begin{tabular}{lll}
\hline & $\begin{array}{l}\text { Zwolan et al. } \\
{[2014](n=18)}\end{array}$ & $\begin{array}{l}\text { Sarant et al. } \\
{[2019]\left(n=59^{\mathrm{a}}\right)}\end{array}$ \\
\hline HUI-3 & & \\
Hearing & $* *$ & $* *$, $/ \mathrm{ns} \sigma^{*}$ \\
Multi-attribute & ns & $*$, $/ \mathrm{ns} \mathrm{\sigma ^{* }}$ \\
\hline
\end{tabular}

${ }^{*} p<0.05 ;{ }^{* *} p<0.01$. ns, not significant $(p>0.05)$;, outcome for female subjects; $\sigma^{x}$, outcome for male subjects.

${ }^{a}$ At baseline, 59 patients were assessed. Out of these, 20 patients were evaluated 18 months postoperatively.

Knopke et al. [2016, 2019] indicated that overall stress perception, assessed by the Perceived Stress Questionnaire (PSQ-30), was low pre- and postoperatively and no additional significant decrease was observed. A significant decrease in the PSQ subscale (lack of) "joy" was found in subjects aged 80 years and older but not in subjects aged 70-80 years. Furthermore, Knopke et al. [2019] showed a significant negative correlation between HRQoL (measured by the NCIQ) and perceived stress in 70- to
80 -year-old subjects, but not in subjects aged 80 years and older. Loneliness scores significantly reduced both 6 months $(p<0.001)$ and 12 months $(p<0.001)$ postoperatively compared to baseline in the study by Contrera et al. [2017]. Sarant et al. [2019] reported an improvement of loneliness, social isolation, anxiety, and depression 18 months postoperatively. However, they did not mention if these improvements were significant.

\section{Activities Outcomes}

Sonnet et al. [2017] demonstrated that the Instrumental Activities of Daily Living (I-ADL) questionnaire's autonomy scores were stable at 6 months and improved significantly at 12 months postimplantation $(p<0.05)$. Sarant et al. [2019] also showed postoperative improvements of everyday and physical activities, but they did not indicate if these improvements were significant.

\section{Risks of Bias}

Six of the accepted studies were at risk of response bias as they reported a response rate of $<80 \%$. Chung et al. [2012], Zwolan et al. [2014], Choi et al. [2016], Contrera et al. [2017], and Contrera et al. [2016] reported total re- 
Table 6. Daily living performance outcomes

\begin{tabular}{|c|c|c|c|c|c|c|c|c|c|}
\hline & $\begin{array}{l}\text { Mosnier } \\
\text { et al. [2015] } \\
(n=94)\end{array}$ & $\begin{array}{l}\text { Castiglione } \\
\text { et al. [2016] } \\
(n=15)\end{array}$ & $\begin{array}{l}\text { Choi et al. } \\
{[2016]} \\
(n=50)\end{array}$ & $\begin{array}{l}\text { Contrera } \\
\text { et al. [2017] } \\
(n=50)\end{array}$ & $\begin{array}{l}\text { Sonnet } \\
\text { et al. [2017] } \\
(n=16)\end{array}$ & $\begin{array}{l}\text { Knopke } \\
\text { et al. [2016] } \\
(n=17)\end{array}$ & $\begin{array}{l}\text { Knopke } \\
\text { et al. [2019] } \\
(n=86)\end{array}$ & $\begin{array}{l}\text { Claes } \\
\text { et al. [2018] } \\
(n=20)\end{array}$ & $\begin{array}{l}\text { Sarant } \\
\text { et al. [2019] } \\
\left(n=59^{\mathrm{a}}\right)\end{array}$ \\
\hline \multicolumn{10}{|l|}{ Mental well-being } \\
\hline \multicolumn{10}{|l|}{ HADS } \\
\hline Anxiety & & & & & & & & $* *, \cup$ & $?$ \\
\hline Depression & & & & & & & & $* *, \cup$ & ? \\
\hline \multicolumn{10}{|l|}{ ADS-L } \\
\hline Total & & & & & & ns & ns & & \\
\hline \multicolumn{10}{|l|}{ GDS } \\
\hline Total & ns & $* *$ & * & & & & & & \\
\hline \multicolumn{10}{|l|}{ HDRS } \\
\hline Total & & & & & ns & & & & \\
\hline Demands & & & & & & ns & $* / \mathrm{ns}^{\mathrm{c}}$ & & \\
\hline Total & & & & & & ns & n.s & & \\
\hline \multicolumn{10}{|l|}{ Loneliness } \\
\hline \multicolumn{10}{|c|}{ UCLA Loneliness Scale } \\
\hline Total & & & & $* * *$, & & & & & \\
\hline \multicolumn{10}{|l|}{ LSNS } \\
\hline Total & & & & & & & & & ? \\
\hline \multicolumn{10}{|l|}{ Loneliness Scale } \\
\hline Total & & & & & & & & & ? \\
\hline \multicolumn{10}{|l|}{ Activities } \\
\hline \multicolumn{10}{|l|}{ Physical activity } \\
\hline \multicolumn{10}{|l|}{ Everyday activities } \\
\hline \multicolumn{10}{|l|}{ Autonomy } \\
\hline $\begin{array}{l}{ }^{*} p<0.05 ; * * p<0 \\
\cup \text { indicates the CI sc } \\
\text { shading indicates not } \\
{ }^{\text {a } A t ~ b a s e l i n e, ~} 59 \mathrm{p} \\
{ }^{\mathrm{b}} \text { Score significant } \\
{ }^{\mathrm{c}} \text { Score significant } \\
{ }^{\mathrm{d}} \text { Score was stable }\end{array}$ & $\begin{array}{l}* * * p<0.001 \\
12 \text { months po } \\
\text { licable; ? indic } \\
\text { tts were assess } \\
\text { nproved in the } \\
\text { aproved in the } \\
\text { months posto }\end{array}$ & $\begin{array}{l}\text { ns, not signifi } \\
\text { toperatively d } \\
\text { tes an improv } \\
\text { ed. Out of the } \\
\text { group over } 80 \\
70 \text { - to } 80 \text {-year } \\
\text { geratively and }\end{array}$ & $\begin{array}{l}\text { t }(p>0.05) \text {. } \\
\text { not significai } \\
\text { hent was obse } \\
20 \text { patients } w \\
\text { ears old but } n \\
\text { ld group at } 1 \\
\text { proved signi }\end{array}$ & $\begin{array}{l}\text { indicates th } \\
\text { tly differ from } \\
\text { ved, but it wa } \\
\text { re evaluated } 1 \\
\text { t in the group } \\
\text { months postc } \\
\text { icantly } 12 \text { mol }\end{array}$ & $\begin{array}{l}\text { outcome ren } \\
\text { the preopera } \\
\text { not mention } \\
8 \text { months pos } \\
\text { aged } 70-80 \text { y } \\
\text { peratively, bu } \\
\text { ths postopera }\end{array}$ & $\begin{array}{l}\text { nained stable } \\
\text { tive score anc } \\
\text { led if the chan } \\
\text { toperatively. } \\
\text { rears. } \\
\text { th not in the } 80 \\
\text { atively. }\end{array}$ & $\begin{array}{l}\text { between } 6 \text { an } \\
\text { the score } 6 \text { r } \\
\text { ge of scores } w \\
0+\text { group. }\end{array}$ & $\begin{array}{l}12 \text { months } p \\
\text { nonths postop } \\
\text { as significant. }\end{array}$ & $\begin{array}{l}\text { ostoperatively } \\
\text { peratively; gray }\end{array}$ \\
\hline
\end{tabular}

sponse rates between 69 and $78 \%$. However, they divided their participants according to age or hearing device (hearing aid or CI) and did not mention the response rate per group. Hence, it was not clear whether response bias occurred in their participants eligible for review. Vermeire et al. [2005] indicated a response rate of 33\%. The questionnaire they used (HHIA) was only introduced to their clinic's CI program after the study had already start- ed. Consequently, a lot of their participants had no preand postoperative HHIA scores.

Selection bias may have affected all included studies because of differences in reimbursement criteria all over the world. Currently, each country individually determines the criteria subjects with hearing impairment must meet to be eligible for CI surgery and no internationally accepted criteria exist. Participants of the eligible studies 
may therefore differ in, for example, psycho-acoustic measures such as pure tone audiometry or speech reception thresholds.

Four studies used suboptimal statistical methods. Sonnet et al. [2017], Castiglione et al. [2016], and Zwolan et al. [2014] all performed parametric tests, while non-parametric tests would have been more appropriate due to their small sample sizes $(n<20)$. In addition, Sonnet et al. [2017] and Park et al. [2011] did not consider the repeated measures per participant and did not use a test for related samples. Post hoc corrections for repeated measures were not mentioned in 7 articles using statistical methods requiring these corrections [Vermeire et al., 2005; Chung et al., 2012; Mosnier et al., 2015; Knopke et al., 2016; Olze et al., 2016; Mosnier et al., 2018; Knopke et al., 2019]. Moreover, the statistical analyses of Noble et al. [2019] and Sarant et al. [2019] were incomplete regarding HRQoL changes pre- and postoperatively. Noble et al. [2009] did not perform statistical analysis to evaluate these changes, only describing it qualitatively. Sarant et al. [2019] did not report statistical analysis of part of their questionnaire data.

Other risks of bias included a small sample size of participants meeting the inclusion criteria of the review $(n<$ 20 ), which was the case in 5 studies [Noble et al., 2009; Zwolan et al., 2014; Castiglione et al., 2016; Knopke et al., 2016; Sonnet et al., 2017]. All studies used either the original version of validated questionnaires or validated translations. Only Park et al. [2011] and Sonnet et al. [2017] mentioned their questionnaire administration method. Park et al. [2011] explained that the questionnaires were self-administered as paper-and-pencil tests, while the HDRS in Sonnet et al. [2017] was administered by an interviewer (psychiatrist). In the other studies the questionnaire administration method was not mentioned. Sarant et al. [2019] stated that the Bayer Activities of Daily Living Scale (B-ADL) was completed by proxy respondents, which means participants did not fill it in themselves. The use of proxy respondents was not mentioned in the other studies.

\section{Discussion}

The aim of this study was to critically assess the current literature on changes in HRQoL in severely hearing-impaired older adult CI users. Studies generally reported a significant improvement of disease-specific HRQoL postoperatively, mainly in studies using hearing-specific or CI-specific questionnaires. In particular, the NCIQ, the most commonly used questionnaire across all studies, showed significant improvements in most subdomains. However, high-level evidence-based medicine is lacking. The improvement of disease-specific HRQoL after improving the hearing function of severely hearing-impaired individuals with a CI was to be expected, as hearing loss is generally associated with lower HRQoL [Ciorba et al., 2012; Contrera et al., 2017]. Nevertheless, currently available objective speech recognition measures are only weakly associated with subjective self-reported HRQoL [McRackan et al., 2018]. Hence, these objective measures cannot capture all aspects of real-life communication and social interaction, both of which are far more challenging than currently available speech-recognition tasks. Generic HRQoL questionnaires' outcomes were less consistent than the hearing-specific questionnaires' outcomes. Therefore, generic HRQoL questionnaires seem to be too general to be able to uncover all the improvements a CI can entail, in line with studies by Patrick and Deyo [1989] and Lin et al. [2012]. They reported that disease-specific questionnaires are more sensitive to possibly important outcome changes than generic questionnaires. A disadvantage of disease-specific questionnaires to assess HRQoL in CI users could be that the questionnaires are only hearing specific. They are not designed to be used across patient populations, diseases, or interventions, which is relevant for economic evaluation of medical interventions. Disease-specific questionnaires specifically developed for use in CI recipients, such as the commonly used NCIQ, lack involvement of CI recipients in the development of item banks for the instruments, which could result in the absence of certain important CI-related HRQoL concepts [McRackan et al., 2017, 2018a, b]. Moreover, the NCIQ was validated using a limited number of participants from only one clinical site and psychometric testing was performed using former, less rigorous techniques compared to current standards. McRackan et al. [2019] therefore created a new HRQoL item bank for CI recipients, based on item response theory, which was used to develop a new CI-specific QoL evaluation instrument, the Cochlear Implant Quality of Life (CIQOL), capturing a broader spectrum of important HRQoL concepts [McRackan et al., 2017, 2019]. Limitations of current hearing-specific questionnaires, such as SSQ12 and APHAB, include their lack of validation in CI recipients. Those instruments are primarily developed and validated for hearing aid users and/or those with mild to moderate hearing loss, and could therefore fail to capture some important HRQoL factors related to cochlear implantation [McRackan et al., 2017, 2018]. Overall, disease-specific 
instruments can be considered relevant in the following 4 cases, according to the European network for Health Technology Assessment (EUnetHTA): (1) to get additional information on HRQoL during the assessment of the risk-benefit of a drug or device for registration purposes; (2) more in-depth assessment of how life is affected precisely if an effect is observed on a generic HRQoL instrument; (3) assessment of the HRQoL benefits of an intervention compared to a relevant comparator for the same indication if no effect on a generic HRQoL instrument is found but a HRQoL benefit is nevertheless assumed; (4) assessment of HRQoL benefits of an intervention if no adequate generic instrument, reflecting the HRQoL dimensions on which change is considered meaningful according to the society, is available [EUnetHTA, 2015]. Consequently, generic and disease-specific HRQoL instruments are rather complementary than contradictory.

The cost-effectiveness of CI is a much-debated topic in clinical practice and research [Bond et al., 2009; Laske et al., 2019]. Quality-adjusted life years (QALYs) is currently the lead metric to evaluate the cost-effectiveness of medical interventions. QALYs can be used to compare the benefits of several treatments and weigh up the costs against the gain in HRQoL over time. It is calculated by multiplying the HRQoL utility score in a health state with the duration of time spent in that health state. Only standardized generic HRQoL questionnaires such as the HUI-3 or EQ-5D can be used to obtain these utility scores [Kind, 1996; Whitehead and Ali, 2010]. The EQ-5D was validated using a random sample of 3,000 UK residents to represent original population preferences. First, the EQ5D-3L was developed with 3 response alternatives or levels in the following 5 domains: mobility, self-care, usual activities, pain/discomfort, and anxiety/depression. Later, EQ-5D-3L turned out to be less responsive and reliable than EQ-5D with 5 levels, so it was replaced by the 5-level version. QALYs are calculated based on generic questionnaires so they are equally insensitive to subtle but potentially important outcomes. However, disease-specific HRQoL questionnaires do not provide utility scores and are often based on a less sophisticated methodology and validation [Lin et al., 2012]. Hence, they cannot be used to calculate QALYs, which may be a limitation depending on the aim of the study. Disadvantages of QALYs involve ethical questions about, for example, the valuation of someone's life over another's and skepticism about the applicability in middle- and low-income countries [Kularatna et al., 2013; Pettitt et al., 2016]. In these countries, disability-adjusted life years (DALYs) could be an alter-

Quality of Life after Cochlear

Implantation in Older Adults native to QALYs. These metrics are a modified version of QALYs measuring health loss due to poor HRQoL instead of health gain due to HRQoL improvement after medical intervention [Grimes et al., 2014; Rios-Diaz et al., 2016].

Overall, various instruments and methods are used to assess HRQoL as no gold standard HRQoL questionnaire currently exists for CI users. The lack of consensus complicates comparisons between studies and reveals the need for a standardized HRQoL study protocol including all relevant generic and disease-specific instruments [Claes et al., 2016]. Researchers and clinicians should not exclusively focus on reliability and validity when selecting HRQoL assessment instruments. Study design, research aims, and combining several HRQoL measures should also be considered. Using supplementary HRQoL questionnaires could be beneficial if one HRQoL questionnaire cannot provide sufficient information to meet study objectives or clinical purposes. However, researchers or clinicians must be familiar with the questionnaires to avoid significant overlap between measures. Comprehensive reporting of all subdomain scores is also recommended to provide a clear overview of the results.

Furthermore, differences in CI reimbursement criteria all over the world may have led to selection bias, because psycho-acoustic measures at baseline could differ between studies conducted in different countries. Reporting individual data points of raw pure tone averages obtained preoperatively per participant is therefore recommended [de Bruijn et al., 2001a, b; Van Rompaey et al., 2009]. That way, a clear overview of the speech reception thresholds and pure tone audiometry thresholds of participants at baseline could be provided and studies could be compared more accurately. Additionally, several excluded studies used the Glasgow Benefit Inventory (GBI) to assess HRQoL postoperatively with questions about their pre- and postoperative HRQoL. Recall of events or states prior to major events could lead to inaccurate or incomplete data according to Choi et al. [2005]. Vermeire et al. [2005] confirm their findings as they reported variations in HRQoL pre- and postoperatively if the participants' preoperative HRQoL is assessed postoperatively. More specifically, GBI scores decreased postoperatively over time in their study, implying HRQoL benefits reduced over time. Vermeire et al. [2005] explained these variations due to the fact that participants can have difficulties in recalling their preoperative state if there is too much time between the operation and the postoperative HRQoL assessment. Hence, studies using GBI or studies assessing pre- and postoperative HRQoL after implantation are at risk of potential bias [Choi and Pak, 2005]. 
As a result, studies using GBI or studies assessing pre- and postoperative HRQoL after implantation were not considered eligible for review. Studies using tinnitus questionnaires were also excluded for review. Tinnitus was considered a disorder of the auditory system, which could have an influence on the HRQoL of patients. Nevertheless, tinnitus questionnaires are not primarily focused on HRQoL assessment and tinnitus presence does not necessarily imply that HRQoL is negatively affected. In contrast, studies have shown that bilateral vestibulopathy, which is a common comorbidity of severe to profound SNHL, has a significant negative effect on HRQoL [Guinand et al., 2012; Sun et al., 2014]. However, the impact of vestibular function on the HRQoL of CI users was not considered in the articles accepted for review.

The use of suboptimal statistical tests and no post hoc correction for repeated measures were common limitations of the statistical analysis of the eligible studies. Therefore, future studies are recommended to use statistical methods appropriate to their data to demonstrate differences in HRQoL pre- and postoperatively as accurately as possible. The administration method could influence the results of questionnaires, but Park et al. [2011] and Sonnet et al. [2017] were the only authors who mentioned the questionnaire administration method in their studies. If an interviewer administered questionnaires, he or she could, for example, consciously or subconsciously influence the participants' responses, whereas self-assessment, for example, could lead to questions not being understood properly [Choi and Pak, 2005]. Proxy respondents could also cause a risk of bias, as they fill in the questionnaires for someone else and may not provide exactly the same responses the participants would provide. The use of proxy respondents was only mentioned by Sarant et al. [2019] for one questionnaire. Either the validated original version of questionnaires or a validated translation of questionnaires was used in all studies. Most HRQoL questionnaires have several validated translations, which enables a comparison between data from several institutes and countries. Using validated questionnaires is crucial to obtain reliable results and is strongly recommended. Response bias was a common limitation as well, because working with an older population could imply a higher dropout rate caused by, for example, a higher risk of death, illness, and so on.

In addition, research exclusively focusing on the assessment of HRQoL in older CI users is limited. Cognition and clinical psycho-acoustic measures are usually the primary outcome measures in literature on these subjects. That is why some articles did not appear in the PubMed and
Cochrane Library search records and had to be included via reference list searching of relevant articles. Hence, there is a chance that relevant literature might have been missed. Another limitation of the review was its retrospective design. It is possible that more studies meeting the inclusion criteria will have been published by the time this review is published. Two full-text articles could not be retrieved and could consequently not be assessed for eligibility.

\section{Conclusion}

A systematic review of the literature was performed on changes in HRQoL before and after cochlear implantation in older adults. An improvement of HRQoL was generally reported when using disease-specific instruments, which are questionnaires designed to detect treatment-specific changes, but high-level evidence-based medicine is lacking. In contrast, the outcomes of generic HRQoL questionnaires - which are used across patient populations, disorders, and interventions - were rather ambiguous. The methodology of HRQoL analysis was found to be heterogeneous for types of instruments used and the individual patients' hearing levels. Future studies should use a standardized, multidimensional, and comprehensive study protocol, including generic and complementary disease-specific HRQoL instruments tailored purposefully to measure HRQoL, utility, and/or daily life performance [Claes et al., 2016].

\section{Statement of Ethics}

The authors have no ethical conflicts to disclose.

\section{Disclosure Statement}

The authors have no conflicts of interest to declare.

\section{Funding Sources}

The Antwerp University Hospital currently receives a research grant from MED-EL (Innsbruck, Austria).

\section{Author Contributions}

E.A. and G.M. performed study selection. E.A. drafted the manuscript. A.G., V.T., O.M.V., P.V.d.H., V.V.R., and G.M. critically revised the manuscript. All authors read and approved the final manuscript.
Andries et al. 


\section{References}

Aimoni C, Ciorba A, Hatzopoulos S, Ramacciotti G, Mazzoli M, Bianchini C, et al. Cochlear implants in subjects over age 65: quality of life and audiological outcomes. Med Sci Monit. 2016 Aug;22:3035-42.

Alhanbali S, Dawes P, Lloyd S, Munro KJ. Selfreported listening-related effort and fatigue in hearing-impaired adults. Ear Hear. 2017 Jan/ Feb;38(1):e39-48.

Ambert-Dahan E, Laouénan C, Lebredonchel M, Borel S, Carillo C, Bouccara D, et al. Evaluation of the impact of hearing loss in adults: validation of a quality of life questionnaire. Eur Ann Otorhinolaryngol Head Neck Dis. 2018 Feb;135(1):25-31.

Angelo TC, Moret AL, Costa OA, Nascimento LT, Alvarenga KF. Quality of life in adult cochlear implant users. CoDAS. 2016 Apr;28(2):10612.

Arnoldner C, Lin VY, Bresler R, Kaider A, Kuthubutheen J, Shipp D, et al. Quality of life in cochlear implantees: comparing utility values obtained through the Medical Outcome Study Short-Form Survey-6D and the Health Utility Index Mark 3. Laryngoscope. 2014a Nov;124(11):2586-90.

Arnoldner C, Lin VY, Honeder C, Shipp D, Nedzelski J, Chen J. Ten-year health-related quality of life in cochlear implant recipients: prospective SF-36 data with SF-6D conversion. Laryngoscope. 2014b Jan;124(1):278-82.

Bond M, Mealing S, Anderson R, Elston J, Weiner G, Taylor RS, et al. The effectiveness and cost-effectiveness of cochlear implants for severe to profound deafness in children and adults: a systematic review and economic model. Health Technol Assess. 2009 Sep 13(44):1-330.

Calvino M, Gavilán J, Sánchez-Cuadrado I, PérezMora R, Muñoz E, Díez-Sebastián J, et al. Using the HISQUI29 to assess the sound quality levels of Spanish adults with unilateral cochlear implants and no contralateral hearing. Eur Arch Otorhinolaryngol. 2016 Sep;273(9) 2343-3.

Capretta NR, Moberly AC. Does quality of life depend on speech recognition performance for adult cochlear implant users? Laryngoscope. 2016 Mar;126(3):699-706.

Castiglione A, Benatti A, Velardita C, Favaro D, Padoan E, Severi D, et al. Aging, Cognitive Decline and Hearing Loss: Effects of Auditory Rehabilitation and Training with Hearing Aids and Cochlear Implants on Cognitive Function and Depression among Older Adults. Audiol Neurotol. 2016;21(1 Suppl 1):21-8.

Cherko M, Hickson L, Bhutta M. Auditory deprivation and health in the elderly. Maturitas. 2016 Jun; 88:52-7.

Choi BC, Pak AW. A catalog of biases in questionnaires. Prev Chronic Dis. 2005 Jan;2(1):A13.

Choi JS, Betz J, Li L, Blake CR, Sung YK, Contrera KJ, et al. Association of Using Hearing Aids or Cochlear Implants With Changes in Depressive Symptoms in Older Adults. JAMA
Otolaryngol Head Neck Surg. 2016 Jul;142(7): 652-7.

Chung J, Chueng K, Shipp D, Friesen L, Chen JM, Nedzelski JM, et al. Unilateral multi-channel cochlear implantation results in significant improvement in quality of life. Otol Neurotol. 2012 Jun;33(4):566-71.

Ciorba A, Bianchini C, Pelucchi S, Pastore A. The impact of hearing loss on the quality of life of elderly adults. Clin Interv Aging. 2012;7:159-63.

Claes AJ, Mertens G, Gilles A, Hofkens-Van den Brandt A, Fransen E, Van Rompaey V, et al. The Repeatable Battery for the Assessment of Neuropsychological Status for Hearing Impaired Individuals (RBANS-H) before and after Cochlear Implantation: A Protocol for a Prospective, Longitudinal Cohort Study. Front Neurosci. 2016 Nov; 10:512.

Claes AJ, Van de Heyning P, Gilles A, Van Rompaey V, Mertens G. Cognitive Performance of Severely Hearing-impaired Older Adults Before and After Cochlear Implantation: Preliminary Results of a Prospective, Longitudinal Cohort Study Using the RBANS-H. Otol Neurotol. 2018 Oct;39(9):e765-73.

Cloutier F, Bussières R, Ferron P, Côté M. OCTO "Outcomes of cochlear implant for the octogenarians: audiologic and quality-of-life". Otol Neurotol. 2014 Jan;35(1):22-8.

Cohen SM, Labadie RF, Dietrich MS, Haynes DS. Quality of life in hearing-impaired adults: the role of cochlear implants and hearing aids. Otolaryngol Head Neck Surg. 2004 Oct; 131(4):413-22.

Contrera KJ, Betz J, Li L, Blake CR, Sung YK, Choi JS, et al. Quality of life after intervention with a cochlear implant or hearing aid. Laryngoscope. 2016 Sep;126(9):2110-5.

Contrera KJ, Sung YK, Betz J, Li L, Lin FR. Change in loneliness after intervention with cochlear implants or hearing aids. Laryngoscope. 2017 Aug;127(8):1885-9.

Cooper HR, Carpenter L, Aleksy W, Booth CL, Read TE, Graham JM, et al. UCH/RNID single channel extracochlear implant: results in thirty profoundly deafened adults. J Laryngol Otol Suppl. 1989;18:22-38.

Damen GW, Beynon AJ, Krabbe PF, Mulder JJ, Mylanus EA. Cochlear implantation and quality of life in postlingually deaf adults: long-term follow-up. Otolaryngol Head Neck Surg. 2007 Apr;136(4):597-604.

de Bruijn AJ, Tange RA, Dreschler WA. Efficacy of evaluation of audiometric results after stapes surgery in otosclerosis. I. The effects of using different audiologic parameters and criteria on success rates. Otolaryngol Head Neck Surg. 2001a Jan;124(1):76-83.

de Bruijn AJ, Tange RA, Dreschler WA. Efficacy of evaluation of audiometric results after stapes surgery in otosclerosis. II. A method for reporting results from individual cases. Otolaryngol Head Neck Surg. 2001b Jan;124(1):84-9.

Derks LS, Wegner I, Smit AL, Thomeer HG, Topsakal V, Grolman W. Effect of day-case uni- lateral cochlear implantation in adults on general and disease-specific quality of life, postoperative complications and hearing results, tinnitus, vertigo and cost-effectiveness: protocol for a randomised controlled trial. BMJ Open. 2016 Oct;6(10):e012219.

Di Nardo W, Anzivino R, Giannantonio S, Schinaia L, Paludetti G. The effects of cochlear implantation on quality of life in the elderly. Eur Arch Otorhinolaryngol. 2014 Jan;271(1):6573.

Djalilian HR, King TA, Smith SL, Levine SC. Cochlear implant in the elderly: Results and quality of life assessment. Otolaryngol Head Neck Surg. 1999;121(2_suppl):P227-8.

Eshraghi AA, Rodriguez M, Balkany TJ, Telischi FF, Angeli S, Hodges AV, et al. Cochlear implant surgery in patients more than seventynine years old. Laryngoscope. 2009 Jun; 119(6):1180-3

EUnetHTA. Endpoints used for relative effectiveness assessment: health-related quality of life and utility measures. 2015.

Francis HW, Chee N, Yeagle J, Cheng A, Niparko JK. Impact of cochlear implants on the functional health status of older adults. Laryngoscope. 2002 Aug;112(8 Pt 1):1482-8.

Francis HW, Yeagle JA, Thompson CB. Clinical and psychosocial risk factors of hearing outcome in older adults with cochlear implants. Laryngoscope. 2015 Mar;125(3):695-702.

Fuller C, Free R, Maat B, Başkent D. Musical background not associated with self-perceived hearing performance or speech perception in postlingual cochlear-implant users. J Acoust Soc Am. 2012 Aug;132(2):100916.

Grimes CE, Henry JA, Maraka J, Mkandawire NC, Cotton M. Cost-effectiveness of surgery in low- and middle-income countries: a systematic review. World J Surg. 2014 Jan;38(1): 252-63.

Guinand N, Boselie F, Guyot JP, Kingma H. Quality of life of patients with bilateral vestibulopathy. Ann Otol Rhinol Laryngol. 2012 Jul; 121(7):471-7.

Hamzavi J, Franz P, Baumgartner WD, Gstöettner W. Tinnitus and cochlear implants. Audiology. 2001 Jan-Feb;40(1):26-31.

Hinder D, Linder TE, Schlegel-Wagner C, Candreia C. [Benefit of Bimodal Stimulation with Cochlear Implant and Hearing Aid in Elderly Patients]. Laryngorhinootologie. 2017 Jul; 96(7):456-60.

Hirschfelder A, Gräbel S, Olze H. The impact of cochlear implantation on quality of life: the role of audiologic performance and variables. Otolaryngol Head Neck Surg. 2008 Mar; 138(3):357-62.

Hofkens-Van den Brandt A, Mertens G, Gilles A, Fransen E, Lassaletta L, Gavilan J, et al. Auditory Performances in Older and Younger Adult Cochlear Implant Recipients: use of the HEARRING Registry. Otol Neurotol. 2019 Sep;40(8):e787-95. 
Horsman J, Furlong W, Feeny D, Torrance G. The Health Utilities Index (HUI): concepts, measurement properties and applications. Health Qual Life Outcomes. 2003 Oct;1(1):54-54.

Huarte A, Lezaun R, Manrique M. Quality of life outcomes for cochlear implantation in the elderly. Audiol Neurotol. 2014;19(Suppl 1):36-9.

Jayakody DM, Friedland PL, Nel E, Martins RN, Atlas MD, Sohrabi HR. Impact of Cochlear Implantation on Cognitive Functions of Older Adults: Pilot Test Results. Otol Neurotol. 2017 Sep;38(8):e289-95.

Kelsall DC, Arnold RJ, Lionnet L. Patient-Reported Outcomes From the United States Clinical Trial for a Hybrid Cochlear Implant. Otol Neurotol. 2017 Oct;38(9):1251-61.

Kelsall DC, Shallop JK, Burnelli T. Cochlear implantation in the elderly. Am J Otol. 1995 Sep; 16(5):609-15.

Kind P. The EuroQol instrument: an index of health-related quality of life. 2nd ed. Philadelphia: Lippincott-Raven; 1996.

Knopke S, Gräbel S, Förster-Ruhrmann U, Mazurek B, Szczepek AJ, Olze H. Impact of cochlear implantation on quality of life and mental comorbidity in patients aged 80 years. Laryngoscope. 2016 Dec;126(12):2811-6.

Knopke S, Häussler S, Gräbel S, Wetterauer D, Ketterer M, Fluger A, et al. Age-Dependent Psychological Factors Influencing the Outcome of Cochlear Implantation in Elderly $\mathrm{Pa}$ tients. Otol Neurotol. 2019 Apr;40(4):e44153.

Knutson JF, Murray KT, Husarek S, Westerhouse K, Woodworth G, Gantz BJ, et al. Psychological change over 54 months of cochlear implant use. Ear Hear. 1998 Jun;19(3):191-201.

Kularatna S, Whitty JA, Johnson NW, Scuffham PA. Health state valuation in low- and middle-income countries: a systematic review of the literature. Value Health. 2013 Sep-Oct; 16(6):1091-9.

Kunimoto M, Yamanaka N, Kimura T, Yoda J, Yokoyama M, Togawa A, et al. The benefit of cochlear implantation in the Japanese elderly. Auris Nasus Larynx. 1999 Apr;26(2):131-7.

Laske RD, Dreyfuss M, Stulman A, Veraguth D, Huber AM, Röösli C. Age Dependent CostEffectiveness of Cochlear Implantation in Adults. Is There an Age Related Cut-off? Otol Neurotol. 2019 Aug;40(7):892-9.

Lenarz T, James C, Cuda D, Fitzgerald O'Connor A, Frachet B, Frijns JH, et al. European multicentre study of the Nucleus Hybrid L24 cochlear implant. Int J Audiol. 2013 Dec;52(12): 838-48.

Liberati A, Altman DG, Tetzlaff J, Mulrow C, Gøtzsche PC, Ioannidis JP, et al. The PRISMA statement for reporting systematic reviews and meta-analyses of studies that evaluate healthcare interventions: explanation and elaboration. BMJ. 2009 Jul;339:b2700.

Lin FR, Yaffe K, Xia J, Xue QL, Harris TB, Purchase-Helzner E, et al.; Health ABC Study Group. Hearing loss and cognitive decline in older adults. JAMA Intern Med. 2013 Feb; 173(4):293-9.
Lin XJ, Lin IM, Fan SY. Methodological issues in measuring health-related quality of life. TzuChi Med J. 2013;25(1):8-12.

Maillet CJ, Tyler RS, Jordan HN. Change in the quality of life of adult cochlear implant patients. Ann Otol Rhinol Laryngol Suppl. 1995 Apr;165:31-48.

Manrique-Huarte R, Calavia D, Huarte Irujo A, Girón L, Manrique-Rodríguez M. Treatment for Hearing Loss among the Elderly: Auditory Outcomes and Impact on Quality of Life. Audiol Neurotol. 2016;21(Suppl 1):29-35.

McRackan TR, Bauschard M, Hatch JL, FrankoTobin E, Droghini HR, Nguyen SA, et al. Meta-analysis of quality-of-life improvement after cochlear implantation and associations with speech recognition abilities. Laryngoscope. 2018a Apr;128(4):982-90.

McRackan TR, Bauschard M, Hatch JL, FrankoTobin E, Droghini HR, Velozo CA, et al. Meta-analysis of Cochlear Implantation Outcomes Evaluated With General Health-related Patient-reported Outcome Measures. Otol Neurotol. 2018b Jan;39(1):29-36.

McRackan TR, Hand BN, Velozo CA, Dubno JR Cochlear Implant Quality of Life Development Consortium. Cochlear Implant Quality of Life (CIQOL): Development of a Profile Instrument (CIQOL-35 Profile) and a Global Measure (CIQOL-10 Global). J Speech Lang Hear Res. 2019 Sep;62(9):3554-63.

McRackan TR, Velozo CA, Holcomb MA, Camposeo EL, Hatch JL, Meyer TA, et al. Use of Adult Patient Focus Groups to Develop the Initial Item Bank for a Cochlear Implant Quality-of-Life Instrument. JAMA Otolaryngol Head Neck Surg. 2017 Oct;143(10):975-82.

Mick P, Amoodi H, Arnoldner C, Shipp D, Friesen L, Lin V, et al. Cochlear implantation in patients with advanced Ménière's disease. Otol Neurotol. 2014 Aug;35(7):1172-8.

Mo B, Lindbaek M, Harris S. Cochlear implants and quality of life: a prospective study. Ear Hear. 2005 Apr;26(2):186-94.

Mosnier I, Bebear JP, Marx M, Fraysse B, Truy E, Lina-Granade G, et al. Improvement of cognitive function after cochlear implantation in elderly patients. JAMA Otolaryngol Head Neck Surg. 2015 May;141(5):442-50.

Mosnier I, Vanier A, Bonnard D, Lina-Granade G, Truy E, Bordure P, et al. Long-Term Cognitive Prognosis of Profoundly Deaf Older Adults After Hearing Rehabilitation Using Cochlear Implants. J Am Geriatr Soc. 2018 Aug;66(8):1553-61.

Noble W, Tyler R, Dunn C, Bhullar N. Hearing handicap ratings among different profiles of adult cochlear implant users. Ear Hear. 2008 Jan;29(1):112-20.

Noble W, Tyler RS, Dunn CC, Bhullar N. Younger- and older-age adults with unilateral and bilateral cochlear implants: speech and spatial hearing self-ratings and performance. Otol Neurotol. 2009 Oct;30(7):921-9.

Olze H, Gräbel S, Förster U, Zirke N, Huhnd LE, Haupt $\mathrm{H}$, et al. Elderly patients benefit from cochlear implantation regarding auditory re- habilitation, quality of life, tinnitus, and stress. Laryngoscope. 2012 Jan;122(1):196203.

Olze H, Gräbel S, Haupt H, Förster U, Mazurek B. Extra benefit of a second cochlear implant with respect to health-related quality of life and tinnitus. Otol Neurotol. 2012 Sep;33(7): 1169-75.

Olze H, Knopke S, Gräbel S, Szczepek AJ. Rapid Positive Influence of Cochlear Implantation on the Quality of Life in Adults 70 Years and Older. Audiol Neurotol. 2016;21(Suppl 1): 43-7.

Olze H, Szczepek AJ, Haupt H, Förster U, Zirke N, Gräbel S, et al. Cochlear implantation has a positive influence on quality of life, tinnitus, and psychological comorbidity. Laryngoscope. 2011 Oct;121(10):2220-7.

Ottaviani F, Iacona E, Sykopetrites V, Schindler A, Mozzanica F. Cross-cultural adaptation and validation of the Nijmegen Cochlear Implant Questionnaire into Italian. Eur Arch Otorhinolaryngol. 2016 Aug;273(8):2001-7.

Padilla Romero MJ, Sainz Quevedo M, Roldán Segura C. [Cochlear implant in postlingual adults with progressive hearing loss]. Acta Otorrinolaringol Esp. 2004 Dec;55(10):45762.

Palmer CS, Niparko JK, Wyatt JR, Rothman M, de Lissovoy G. A prospective study of the costutility of the multichannel cochlear implant. Arch Otolaryngol Head Neck Surg. 1999 Nov; 125(11):1221-8.

Pan T, Tyler RS, Ji H, Coelho C, Gehringer AK, Gogel SA. Changes in the tinnitus handicap questionnaire after cochlear implantation. Am J Audiol. 2009 Dec;18(2):144-51.

Park E, Shipp DB, Chen JM, Nedzelski JM, Lin VY. Postlingually deaf adults of all ages derive equal benefits from unilateral multichannel cochlear implant. J Am Acad Audiol. 2011 Nov-Dec;22(10):637-43.

Patrick DL, Deyo RA. Generic and disease-specific measures in assessing health status and quality of life. Med Care. 1989 Mar;27(3 Suppl):S217-32.

Pettitt D, Raza S, Naughton B, Roscoe A, Ramakrishnan A, Ali A, et al. The limitations of QALY: a literature review. J Stem Cell Res Ther. 2016;6(4):1000334.

Ramakers GG, Smulders YE, van Zon A, Kraaijenga VJ, Stegeman I, Van Zanten GA, et al. Agreement between health utility instruments in cochlear implantation. Clin Otolaryngol. 2016 Dec;41(6):737-43.

Ramos A, Guerra-Jiménez G, Rodriguez C, Borkoski S, Falcón JC, Perez D. Cochlear implants in adults over 60: a study of communicative benefits and the impact on quality of life. Cochlear Implants Int. 2013 Nov; 14(5): 241-5.

Ramos-Macías Á, Falcón González JC, BorkoskiBarreiro SA, Ramos de Miguel Á, Batista DS, Pérez Plasencia D. Health-Related Quality of Life in Adult Cochlear Implant Users: A Descriptive Observational Study. Audiol Neurotol. 2016;21 Suppl 1:36-42. 
Ramsden JD, Gordon K, Aschendorff A, Borucki L, Bunne M, Burdo S, et al. European Bilateral Pediatric Cochlear Implant Forum consensus statement. Otol Neurotol. 2012 Jun; 33(4):561-5.

Rapport F, Bierbaum M, McMahon C, Boisvert I, Lau A, Braithwaite J, et al. Qualitative, multimethod study of behavioural and attitudinal responses to cochlear implantation from the patient and healthcare professional perspective in Australia and the UK: study protocol. BMJ Open. 2018 May;8(5):e019623.

Rembar S, Lind O, Arnesen H, Helvik AS. Effects of cochlear implants: a qualitative study. Cochlear Implants Int. 2009 Dec;10(4):179-97.

Rios-Diaz AJ, Lam J, Ramos MS, Moscoso AV, Vaughn P, Zogg CK, et al. Global Patterns of QALY and DALY Use in Surgical Cost-Utility Analyses: A Systematic Review. PLoS One. 2016 Feb;11(2):e0148304.

Roland JT Jr, Cosetti M, Wang KH, Immerman S, Waltzman SB. Cochlear implantation in the very young child: long-term safety and efficacy. Laryngoscope. 2009 Nov;119(11):220510.

Roland JT Jr, Gantz BJ, Waltzman SB, Parkinson AJ. Long-term outcomes of cochlear implantation in patients with high-frequency hearing loss. Laryngoscope. 2018 Aug; 128(8): 1939-45.

Rösli M, Hoth S, Baumann I, Praetorius M, Plinkert PK. The impact of cochlear implants on the quality of life of patients with singlesided deafness. HNO. 2015 Mar;63(3):182-8. German.

Rumeau C, Frère J, Montaut-Verient B, Lion A, Gauchard G, Parietti-Winkler C. Quality of life and audiologic performance through the ability to phone of cochlear implant users. Eur Arch Otorhinolaryngol. 2015 Dec;272(12): 3685-92.

Runge CL, Henion K, Tarima S, Beiter A, Zwolan TA. Clinical Outcomes of the Cochlear ${ }^{\mathrm{TM}} \mathrm{Nu}-$ cleus $\left({ }^{\circledR}\right) 5$ Cochlear Implant System and SmartSound ${ }^{\mathrm{TM}} 2$ Signal Processing. J Am Acad Audiol. 2016 Jun;27(6):425-40.

Sanchez-Cuadrado I, Gavilan J, Perez-Mora R, Muñoz E, Lassaletta L. Reliability and validity of the Nijmegen Cochlear Implant Questionnaire in Spanish. Eur Arch Otorhinolaryngol. 2015 Jul;272(7):1621-5.
Sanchez-Cuadrado I, Lassaletta L, Perez-Mora RM, Zernotti M, Di Gregorio MF, Boccio C, et al. Is there an age limit for cochlear implantation? Ann Otol Rhinol Laryngol. 2013 Apr; 122(4):222-8

Sarant J, Harris D, Busby P, Maruff P, Schembri A, Dowell R, et al. The effect of cochlear implants on cognitive function in older adults: initial baseline and 18-month follow up results for a prospective international longitudinal study. Front Neurosci. 2019 Aug;13: 789.

Semenov YR, Martinez-Monedero R, Niparko JK. Cochlear implants: clinical and societal outcomes. Otolaryngol Clin North Am. 2012 Oct;45(5):959-81.

Shin YJ, Deguine O, Valès O, Laborde ML, Fraysse B. Cochlear implants in the elderly. Rev Laryngol Otol Rhinol. 1997;118(4):227-31. French.

Shin YJ, Fraysse B, Deguine O, Valès O, Laborde ML, Bouccara D, et al. Benefits of cochlear implantation in elderly patients. Otolaryngol Head Neck Surg. 2000 Apr;122(4):602-6.

Sladen DP, Zappler A. Older and younger adult cochlear implant users: speech recognition in quiet and noise, quality of life, and music perception. Am J Audiol. 2015 Mar;24(1):31-9.

Sonnet MH, Montaut-Verient B, Niemier JY, Hoen M, Ribeyre L, Parietti-Winkler C. Cognitive Abilities and Quality of Life After Cochlear Implantation in the Elderly. Otol Neurotol. 2017 Sep;38(8):e296-301.

Speers A, George A, Toner J. An aging concern: A retrospective study comparing the audiological and speech outcome measures along with the surgical and quality-of-life outcomes in a group of geriatric patients with those of an adult control group. Cochlear Implants Int. 2015 Jan;16(sup1 Suppl 1):S3-5.

Summerfield A, Barton GR; UK Cochlear Implant Study Group. Sensitivity of EQ-5D-3L, HUI2, HUI3, and SF-6D to changes in speech reception and tinnitus associated with cochlear implantation. Qual Life Res. 2019 May; 28(5):1145-54.

Summerfield AQ, Marshall DH. Preoperative predictors of outcomes from cochlear implantation in adults: performance and quality of life. Ann Otol Rhinol Laryngol Suppl. 1995 Sep; $166: 105-8$

Sun DQ, Ward BK, Semenov YR, Carey JP, Della Santina CC. Bilateral vestibular deficiency: quality of life and economic implications. JAMA Otolaryngol Head Neck Surg. 2014 Jun;140(6):527-34.
Sung YK, Li L, Blake C, Betz J, Lin FR. Association of hearing loss and loneliness in older adults. J Aging Health. 2016 Sep;28(6):979-94.

Tang L, Thompson CB, Clark JH, Ceh KM, Yeagle JD, Francis HW. Rehabilitation and psychosocial determinants of cochlear implant outcomes in older adults. Ear Hear. 2017 Nov/ Dec;38(6):663-71.

Teschner M, Polite C, Lenarz T, Lustig L. Cochlear implantation in different health-care systems: disparities between Germany and the United States. Otol Neurotol. 2013 Jan;34(1): 66-74.

Theofilou P. Quality of life: definition and measurement. Eur J Psychol. 2013;9(1):150-62.

Van Rompaey V, Yung M, Claes J, Häusler R, Martin C, Somers T, et al. Prospective effectiveness of stapes surgery for otosclerosis in a multicenter audit setting: feasibility of the Common Otology Database as a benchmark database. Otol Neurotol. 2009 Dec;30(8): 1101-10.

Vermeire K, Brokx JP, Wuyts FL, Cochet E, Hofkens A, Van de Heyning PH. Quality-oflife benefit from cochlear implantation in the elderly. Otol Neurotol. 2005 Mar;26(2):18895.

Völter C, Götze L, Dazert S, Falkenstein M, Thomas JP. Can cochlear implantation improve neurocognition in the aging population? Clin Interv Aging. 2018 Apr; 13:701-12.

Waltzman SB, Cohen NL, Shapiro W. The benefits of cochlear implantation in the geriatric population. Otolaryngol Head Neck Surg. 1993;108(4): 329-33.

Whitehead SJ, Ali S. Health outcomes in economic evaluation: the QALY and utilities. Br Med Bull. 2010;96(1):5-21.

WHO. The World Health Organization Quality of Life Assessment (WHOQOL): development and general psychometric properties. Soc Sci Med. 1998 Jun;46(12):1569-85.

WHO. WHO global estimates on prevalence of hearing loss. Prevention of Deafness. Geneva: World Health Organisation; 2018.

Yang Z, Cosetti M. Safety and outcomes of cochlear implantation in the elderly: A review of recent. J Otol. 2016 Mar;11(1):1-6.

Zwolan TA, Henion K, Segel P, Runge C. The role of age on cochlear implant performance, use, and health utility: a multicenter clinical trial. Otol Neurotol. 2014 Oct;35(9):1560-8. 